\title{
Light cluster production in intermediate energy heavy-ion collisions induced by neutron-rich nuclei
}

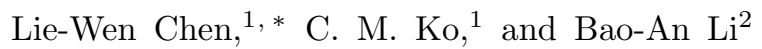 \\ ${ }^{1}$ Cyclotron Institute and Physics Department, Texas A $6 M$ University, College Station, Texas 77843-3366 \\ ${ }^{2}$ Department of Chemistry and Physics, P.O. Box 419, \\ Arkansas State University, State University, Arkansas 72467-0419
}

(Dated: October 31, 2018)

\begin{abstract}
The coalescence model based on nucleon distribution functions from an isospin-dependent transport model is used to study the production of deuteron, triton, and ${ }^{3} \mathrm{He}$ from heavy-ion collisions induced by neutron-rich nuclei at intermediate energies. It is found that the emission time of these light clusters depends on their masses. For clusters with same momentum per nucleon, heavier ones are emitted earlier. Both the yield and energy spectrum of light clusters are sensitive to the density dependence of nuclear symmetry energy, with more light clusters produced in the case of a stiff symmetry energy. On the other hand, effects due to the stiffness of the isoscalar part of nuclear equation of state and the medium dependence of nucleon-nucleon cross sections on light cluster production are unimportant. We have also studied the correlation functions of clusters, and they are affected by the density dependence of nuclear symmetry energy as well, with the stiff symmetry energy giving a stronger anti-correlation of light clusters, particularly for those with large kinetic energies. Dependence of light cluster production on the centrality and incident energy of heavy ion collisions as well as the mass of the reaction system is also investigated.
\end{abstract}

PACS numbers: 25.70.Pq, 21.30.Fe, 21.65.+f, 24.10.Lx

\section{INTRODUCTION}

The equation of state (EOS) of an asymmetric nuclear matter with unequal numbers of protons and neutrons depends crucially on the nuclear symmetry energy. Although the nuclear symmetry energy at normal nuclear matter density is known to be around $30 \mathrm{MeV}$ from the empirical liquid-drop mass formula 1, 2], its values at other densities are poorly known. Studies based on various theoretical models also give widely different predictions 3]. Lack of this knowledge has hampered our understanding of both the structure of radioactive nuclei [4, 5, 6, 7] and many important issues in nuclear astrophysics $8,9,10]$, such as the nucleosynthesis during the pre-supernova evolution of massive stars and the properties of neutron stars [8, 10]. However, recent advances in radioactive nuclear beam facilities provides a unique opportunity to study the density dependence of nuclear symmetry energy [3, 11, 12, 13]. Theoretical studies have already shown that in heavy-ion collisions induced by neutron-rich nuclei, the effect of nuclear symmetry energy can be studied via the pre-equilibrium neutron/proton ratio [14], the isospin fractionation [15, 16, 17, 18, 19], the isoscaling in multifragmentation [20], the proton differential elliptic flow 21, the neutron-proton differential transverse flow [22, 23], and the $\pi^{-}$to $\pi^{+}$ratio [24]. Also, it was found in a recent work that the strength of the correlation function for nucleon pairs with high total momenta in heavy-ion collisions induced by neutron-rich

\footnotetext{
* On leave from Department of Physics, Shanghai Jiao Tong University, Shanghai 200030, China
}

nuclei is sensitive to the nuclear symmetry energy [25]. This result demonstrates that the space-time structure of neutron and proton emission functions is affected by the density dependence of nuclear symmetry energy. Since deuteron production in heavy-ion collisions depends on the space-time and momentum distributions of neutrons and protons at freeze out [26], its production as well as that of other light clusters such as triton and ${ }^{3} \mathrm{He}$ are also expected to be sensitive to the density dependence of nuclear symmetry energy.

To study this quantitatively, we have used in Ref. 27] a coalescence model for treating cluster production from an isospin-dependent Boltzmann-UehlingUhlenbeck (IBUU) transport model. We found that both the multiplicities and energy spectra of light clusters are indeed sensitive to the density dependence of nuclear symmetry energy, with the stiff symmetry energy giving a larger yield of clusters. In particular, the $t /{ }^{3} \mathrm{He}$ ratio at high kinetic energies show the strongest dependence on the stiffness of nuclear symmetry energy. It was also pointed out in Ref. 27] that the effects due to the isospin-independent part of nuclear equation of state and the medium dependence of nucleon-nucleon cross sections are unimportant for light cluster production in heavy ion collisions induced by neutron-rich nuclei. In the present paper, we describe in detail the model used for studying light cluster production in heavy-ion collisions and present also additional results. In particular, we show the correlation functions of these light clusters and the dependence of light cluster production on the centrality and incident energy of heavy ion collisions as well as the mass of the reaction system.

The paper is organized as follows. In Section III we describe the coalescence model for light clus- 
ter production from the nucleon phase-space distributions at freeze out. This includes the construction of the Wigner phase-space density functions for deuteron, triton, and ${ }^{3} \mathrm{He}$. The isospin-dependent BoltzmannUehling-Uhlenbeck (IBUU) transport model used for modeling heavy ion collisions is briefly described in Section III Using the coalescence model given in the previous section, the IBUU model is first applied to study light cluster production in heavy ion collisions involving symmetric nuclei, and the results are compared with available experimental data. Results on light cluster production from heavy-ion collisions induced by neutron-rich nuclei at intermediate energies are presented in Section [V] We show not only the multiplicities and energy spectra of light clusters but also their dependence on the stiffness of nuclear symmetry energy. Dependence of light cluster production on the impact parameter, incident energy, and masses of the reaction system is also given. In Section $\nabla$ we study correlations of light clusters and their dependence on nuclear symmetry energy. We then conclude with a summary and outlook in Section DT

\section{CLUSTER PRODUCTION FROM NUCLEON COALESCENCE}

Light cluster production has been extensively studied in experiments involving heavy ion collisions at all energies (e.g., see Ref. 28] for a recent review). A popular model for describing the production of light clusters in these collisions is the coalescence model (e.g., see Ref. [29] for a theoretical review), which has been used at both intermediate 30, 31, 32] and high energies 33, 34]. In most applications of the coalescence model, the energy spectra for clusters are simply given by the product of the spectra of their constituent nucleons multiplied by an empirical coalescence factor. In Ref. [35], for example, this simplified coalescence model based on the nucleon distributions obtained from the intra-nuclear cascade model gives a satisfactory description of measured high energy spectra of light clusters in intermediate energy (about $100 \mathrm{MeV} /$ nucleon) heavy ion collisions. In more sophisticated coalescence model, the coalescence factor is computed from the overlap of the cluster Wigner phase-space density with the nucleon phase-space distributions at freeze out. The coalescence factor obtained from light particle emission spectra thus provides important information about the space-time structure of nucleon emission source and is thus a sensitive probe to the dynamics of heavy ion collisions [36, 37, 38, 39, 40, 41]. In the present study, this more microscopic coalescence model is adopted for studying light cluster production from heavy ion collisions induced by neutron-rich nuclei at intermediate energies.

\section{A. The coalescence model}

In the coalescence model, the probability for producing a cluster of nucleons is determined by the overlap of its Wigner phase-space density with the nucleon phasespace distributions at freeze out. For a system containing $A$ nucleons, the momentum distribution of a $M$-nucleon cluster with $Z$ protons can be expressed as 33]

$$
\begin{aligned}
& \frac{d N_{M}}{d^{3} K}=G\left(\begin{array}{c}
A \\
M
\end{array}\right)\left(\begin{array}{c}
M \\
Z
\end{array}\right) \frac{1}{A^{M}} \int\left[\prod_{i=1}^{Z} f_{p}\left(\mathbf{r}_{i}, \mathbf{k}_{i}\right)\right] \\
& \times\left[\prod_{i=Z+1}^{M} f_{n}\left(\mathbf{r}_{i}, \mathbf{k}_{i}\right)\right] \rho^{W}\left(\mathbf{r}_{i_{1}}, \mathbf{k}_{i_{1}} \cdots \mathbf{r}_{i_{M-1}}, \mathbf{k}_{i_{M-1}}\right) \\
& \times \delta\left(\mathbf{K}-\left(\mathbf{k}_{1}+\cdots+\mathbf{k}_{M}\right)\right) d \mathbf{r}_{1} d \mathbf{k}_{1} \cdots d \mathbf{r}_{M} d \mathbf{k}_{M} .
\end{aligned}
$$

where $f_{n}$ and $f_{p}$ are, respectively, the neutron and proton phase-space distribution functions at freeze-out; $\rho^{W}$ is the Wigner phase-space density of the $M$-nucleon cluster; $\mathbf{r}_{i_{1}}, \cdots, \mathbf{r}_{i_{M-1}}$ and $\mathbf{k}_{i_{1}}, \cdots, \mathbf{k}_{i_{M-1}}$ are, respectively, the $M-1$ relative coordinates and momenta taken at equal time in the $M$-nucleon rest frame; and $G$ is the spin-isospin statistical factor for the cluster. In transport model simulations for heavy ion collisions, the multiplicity of a $M$-nucleon cluster is then given by [33]

$$
\begin{aligned}
N_{M} & =G \int \sum_{i_{1}>i_{2}>\ldots>i_{M}} d \mathbf{r}_{i_{1}} d \mathbf{k}_{i_{1}} \cdots d \mathbf{r}_{i_{M-1}} d \mathbf{k}_{i_{M-1}} \\
& \times\left\langle\rho_{i}^{W}\left(\mathbf{r}_{i_{1}}, \mathbf{k}_{i_{1}} \cdots \mathbf{r}_{i_{M-1}}, \mathbf{k}_{i_{M-1}}\right)\right\rangle .
\end{aligned}
$$

In the above, $\langle\cdots\rangle$ denotes event averaging and the sum runs over all possible combinations of $M$ nucleons containing $Z$ protons. The spin-isospin statistical factor $G$ is $3 / 8$ for deuteron and $1 / 3$ for triton or ${ }^{3} \mathrm{He}$, with the latter including the possibility of coalescence of a deuteron with another nucleon to form a triton or ${ }^{3} \mathrm{He}$ [2].

As pointed out in Ref. [33], the validity of coalescence model is based on the assumption that nucleon emissions are statistically independent and binding energies of formed clusters as well as the quantum dynamics only play minor roles. Since the binding energies of deuteron, triton and ${ }^{3} \mathrm{He}$ are $2.2 \mathrm{MeV}, 7.72 \mathrm{MeV}$ and $8.48 \mathrm{MeV}$, respectively, the coalescence model for the production of these light clusters in heavy-ion collisions is thus applicable if the colliding system or the emission source has excitation energy per nucleon or temperature above $\sim 9$ $\mathrm{MeV}$. Furthermore, the coalescence model is a perturbative approach and is valid only if the number of clusters formed in the collisions is small. As shown later, this condition is indeed satisfied for energetic deuterons, tritons, and ${ }^{3} \mathrm{He}$ in the collisions we are considering.

In the following, we construct the Wigner phase-space densities for deuteron, triton, and ${ }^{3} \mathrm{He}$. The method can be generalized, although more involved, to the Wigner phase-space density for four-nucleon clusters (e.g., ${ }^{4} \mathrm{He}$ ) and $M$-nucleon clusters. 


\section{B. Wigner phase-space density of deuteron}

For deuterons, we obtain their Wigner phase-space density from the Hulthén wave function [43], i.e.,

$$
\phi(r)=\sqrt{\frac{\alpha \beta(\alpha+\beta)}{2 \pi(\alpha-\beta)^{2}}} \frac{\exp (-\alpha r)-\exp (-\beta r)}{r}
$$

with the parameters $\alpha=0.23 \mathrm{fm}^{-1}$ and $\beta=1.61 \mathrm{fm}^{-1}$ to reproduce the measured deuteron root-mean-square radius of $1.96 \mathrm{fm}$ [4]. The Wigner phase-space density of deuteron can then be obtained from

$$
\rho_{d}^{W}(\mathbf{r}, \mathbf{k})=\int \phi\left(\mathbf{r}+\frac{\mathbf{R}}{2}\right) \phi^{*}\left(\mathbf{r}-\frac{\mathbf{R}}{2}\right) \exp (-i \mathbf{k} \cdot \mathbf{R}) d \mathbf{R}
$$

where $\mathbf{k}=\left(\mathbf{k}_{1}-\mathbf{k}_{2}\right) / 2$ is the relative momentum while $\mathbf{r}=\left(\mathbf{r}_{1}-\mathbf{r}_{2}\right)$ is the relative coordinate of proton and neutron in the deuteron. The $\rho_{d}^{W}(\mathbf{r}, \mathbf{k})$ cannot be derived analytically if one uses Eq. (3). As in Ref. [33], we express the Hulthén wave function in terms of the sum of 15 Gaussian wave functions

$$
\phi(r)=\sum_{i=1}^{15} c_{i}\left(\frac{2 w_{i}}{\pi}\right)^{3 / 4} \exp \left(-w_{i} r^{2}\right)
$$

where the coefficient $c_{i}$ and the width parameter $w_{i}$ in the Gaussian wave function are determined by least square fit, and they are given in Table I With the above deuteron wave function, $\rho_{d}^{W}(\mathbf{r}, \mathbf{k})$ can be obtained analytically as $[33,45]$

$$
\begin{aligned}
& \rho_{d}^{W}(\mathbf{r}, \mathbf{k})=8 \sum_{i=1}^{15} c_{i}^{2} \exp \left(-2 w_{i} r^{2}-\frac{k^{2}}{2 w_{i}}\right) \\
& +16 \sum_{i>j}^{15} c_{i} c_{j}\left(\frac{4 w_{i} w_{j}}{\left(w_{i}+w_{j}\right)^{2}}\right)^{3 / 4} \exp \left(-\frac{4 w_{i} w_{j}}{w_{i}+w_{j}} r^{2}\right) \\
& \times \exp \left(-\frac{k^{2}}{w_{i}+w_{j}}\right) \times \cos \left(2 \frac{w_{i}-w_{j}}{w_{i}+w_{j}} \mathbf{r} \cdot \mathbf{k}\right) .
\end{aligned}
$$

The expansion in Eq. (5) approximates the Hulthén wave function not only in coordinate space but also in momentum space. In momentum space, the normalized Hulthén wave function is given by

$$
\phi(k)=\frac{\sqrt{(\alpha+\beta)^{3} \alpha \beta}}{\pi\left(\alpha^{2}+k^{2}\right)\left(\beta+k^{2}\right)} .
$$

In Fig. 11 we show the exact (solid lines) and fitted (dashed lines) Hulthén wave function in both the coordinate and the momentum space. It is seen that the sum of 15 Gaussian wave functions reproduces satisfactorily the exact Hulthén wave function both in coordinate and momentum spaces.
TABLE I: Gaussian fit coefficient $c_{i}$ and $w_{i}$ to deuteron wave function.

\begin{tabular}{lcr}
\hline \hline$i$ & $c_{i}$ & $w_{i}\left(1 / \mathrm{fm}^{2}\right)$ \\
\hline 1 & $3.49665 \mathrm{E}-1$ & $1.57957 \mathrm{E}-2$ \\
2 & $1.85419 \mathrm{E}-1$ & $3.94293 \mathrm{E}-2$ \\
3 & $1.72279 \mathrm{E}-1$ & $8.99793 \mathrm{E}-2$ \\
4 & $4.62152 \mathrm{E}-2$ & $9.75943 \mathrm{E}-2$ \\
5 & $1.49458 \mathrm{E}-1$ & $1.80117 \mathrm{E}-1$ \\
6 & $7.74205 \mathrm{E}-2$ & $1.93353 \mathrm{E}-1$ \\
7 & $1.48268 \mathrm{E}-4$ & $1.99811 \mathrm{E}-1$ \\
8 & $7.35549 \mathrm{E}-3$ & $2.17921 \mathrm{E}-1$ \\
9 & $4.89047 \mathrm{E}-2$ & $2.89902 \mathrm{E}-1$ \\
10 & $4.19816 \mathrm{E}-2$ & $4.70739 \mathrm{E}-1$ \\
11 & $1.72670 \mathrm{E}-2$ & $4.89604 \mathrm{E}-1$ \\
12 & $1.06294 \mathrm{E}-1$ & $9.27621 \mathrm{E}-1$ \\
13 & $2.51462 \mathrm{E}-4$ & $1.98822 \mathrm{E}+0$ \\
14 & $3.22947 \mathrm{E}-2$ & $2.59243 \mathrm{E}+0$ \\
15 & $1.15826 \mathrm{E}-2$ & $1.44639 \mathrm{E}+1$ \\
\hline \hline
\end{tabular}
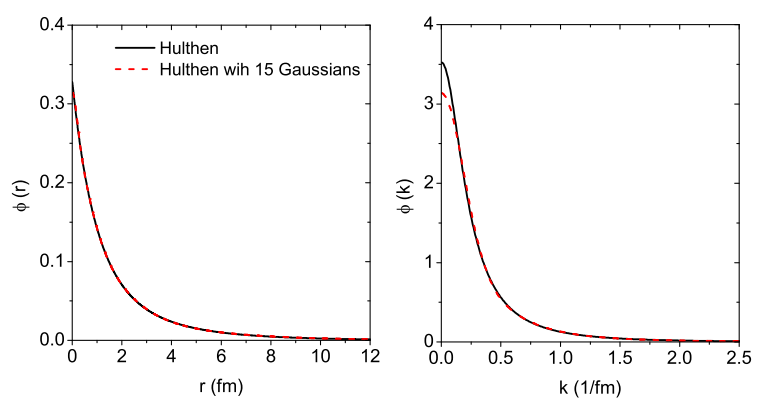

FIG. 1: The exact (solid curves) and fitted (dashed curves) Hulthén wave function in coordinate space (left panel) and momentum space (right panel).

\section{Wigner phase-space densities of triton and ${ }^{3} \mathrm{He}$}

For triton and ${ }^{3} \mathrm{He}$, their Wigner phase-space densities are obtained from their internal wave functions, which are taken to be those of a spherical harmonic oscillator [41, 46], i.e.,

$$
\psi\left(\mathbf{r}_{1}, \mathbf{r}_{2}, \mathbf{r}_{3}\right)=\left(3 \pi^{2} b^{4}\right)^{-3 / 4} \exp \left(-\frac{\rho^{\mathbf{2}}+\lambda^{\mathbf{2}}}{2 b^{2}}\right)
$$

In the above, we have used the normal Jacobi coordinates for a three-particle system, i.e.,

$$
\left(\begin{array}{c}
\mathbf{R} \\
\rho \\
\lambda
\end{array}\right)=J\left(\begin{array}{l}
\mathbf{r}_{1} \\
\mathbf{r}_{2} \\
\mathbf{r}_{3}
\end{array}\right)
$$

where $\mathbf{R}$ is the center-of-mass coordinate, while $\rho$ and $\lambda$ are relative coordinates. The Jacobian matrix is given 
by

$$
J=\left(\begin{array}{ccc}
\frac{1}{3} & \frac{1}{3} & \frac{1}{3} \\
\frac{1}{\sqrt{2}} & -\frac{1}{\sqrt{2}} & 0 \\
\frac{1}{\sqrt{6}} & \frac{1}{\sqrt{6}} & -\frac{2}{\sqrt{6}}
\end{array}\right) .
$$

¿From Eqs. (9) and (10), one obtains $d \mathbf{r}_{1} d \mathbf{r}_{2} d \mathbf{r}_{3}=$ $1 /|J|^{3} d \mathbf{R} d \rho d \lambda=3^{3 / 2} d \mathbf{R} d \rho d \lambda$. It is then easy to check that the wave function Eq. [8] is normalized to one. ¿From the relation $\left(\mathbf{r}_{1}-\mathbf{R}\right)^{2}+\left(\mathbf{r}_{2}-\mathbf{R}\right)^{2}+\left(\mathbf{r}_{3}-\mathbf{R}\right)^{\mathbf{2}}=$ $\rho^{\mathbf{2}}+\lambda^{\mathbf{2}}$, the root-mean-square radius $r_{\text {rms }}$ of triton or ${ }^{3} \mathrm{He}$ can be written as

$$
r_{\mathrm{rms}}^{2}=\int \frac{\rho^{2}+\lambda^{2}}{3}\left|\psi\left(\mathbf{r}_{1}, \mathbf{r}_{2}, \mathbf{r}_{3}\right)\right|^{2} 3^{3 / 2} d \rho d \lambda=b^{2} .
$$

The parameter $b$ is determined to be $1.61 \mathrm{fm}$ and 1.74 fm for triton and ${ }^{3} \mathrm{He}$, respectively, from their measured root-mean-square radii [47].

The Wigner phase-space densities for triton and ${ }^{3} \mathrm{He}$ is then given by

$$
\begin{aligned}
& \rho_{\mathrm{t}\left({ }^{3} \mathrm{He}\right)}^{W}\left(\rho, \lambda, \mathbf{k}_{\rho}, \mathbf{k}_{\lambda}\right) \\
= & \int \psi\left(\rho+\frac{\mathbf{R}_{\mathbf{1}}}{2}, \lambda+\frac{\mathbf{R}_{\mathbf{2}}}{2}\right) \psi^{*}\left(\rho-\frac{\mathbf{R}_{\mathbf{1}}}{2}, \lambda-\frac{\mathbf{R}_{\mathbf{2}}}{2}\right) \\
& \times \exp \left(-i \mathbf{k}_{\rho} \cdot \mathbf{R}_{\mathbf{1}}\right) \exp \left(-i \mathbf{k}_{\lambda} \cdot \mathbf{R}_{\mathbf{2}}\right) 3^{3 / 2} d \mathbf{R}_{\mathbf{1}} d \mathbf{R}_{\mathbf{2}} \\
= & 8^{2} \exp \left(-\frac{\rho^{\mathbf{2}}+\lambda^{\mathbf{2}}}{b^{2}}\right) \exp \left(-\left(\mathbf{k}_{\rho}^{2}+\mathbf{k}_{\lambda}^{2}\right) b^{2}\right),
\end{aligned}
$$

where $\mathbf{k}_{\rho}$ and $\mathbf{k}_{\lambda}$ are relative momenta, which together with the total momentum $\mathbf{K}$ are defined by

$$
\left(\begin{array}{c}
\mathbf{K} \\
\mathbf{k}_{\rho} \\
\mathbf{k}_{\lambda}
\end{array}\right)=J^{-,+}\left(\begin{array}{c}
\mathbf{k}_{1} \\
\mathbf{k}_{2} \\
\mathbf{k}_{3}
\end{array}\right),
$$

with $\mathbf{k}_{1}, \mathbf{k}_{2}$, and $\mathbf{k}_{3}$ being momenta of the three nucleons. The matrix $J^{-,+}$denotes the inverse of the conjugate complex transposition of the Jacobian matrix $J$, i.e.,

$$
J^{-,+}=\left(\begin{array}{ccc}
1 & 1 & 1 \\
\frac{1}{\sqrt{2}} & -\frac{1}{\sqrt{2}} & 0 \\
\frac{1}{\sqrt{6}} & \frac{1}{\sqrt{6}} & -\frac{2}{\sqrt{6}}
\end{array}\right) .
$$

\section{IBUU MODEL AND LIGHT CLUSTER PRODUCTION}

To determine the space-time and momentum distributions of nucleons at freeze out in intermediateenergy heavy ion collisions, we use the isospin-dependent Boltzmann-Uehling-Uhlenbeck (IBUU) transport model [14, 22, 24, 48]. For a review of the IBUU model, we refer the reader to Ref. 12]. The IBUU model includes explicitly the isospin degrees of freedom through different proton and neutron initial distributions as well as their different mean-field potentials and two-body collisions in subsequent dynamic evolutions. For nucleonnucleon cross sections, we use as default the experimental values in free space. To study the effects due to the isospin-dependence of in-medium nucleon-nucleon cross sections $\sigma_{\text {medium }}$, we also use a parameterization obtained from the Dirac-Brueckner approach based on the Bonn A potential [49]. For the experimental free-space cross sections, the neutron-proton cross section is about a factor of 3 larger than the neutron-neutron or proton-proton cross section. On the other hand, the in-medium nucleonnucleon cross sections used here have smaller values and weaker isospin dependence than $\sigma_{\exp }$ but strong density dependence. For the isoscalar potential, we use as default the Skyrme potential with an incompressibility $K_{0}=380$ $\mathrm{MeV}$. This potential has been shown to reproduce the transverse flow data from heavy ion collisions equally well as a momentum-dependent soft potential with $K_{0}=210$ $\mathrm{MeV}$ [50, 51].

The IBUU model is solved with the test particle method 52]. Although the mean-field potential is evaluated with test particles, only collisions among nucleons in each event are allowed. Light cluster production from coalescence of nucleons is treated similar as nucleon-nucleon collisions, i.e., only nucleons in the same event are allowed to coalesce to light clusters. Results presented in the following are obtained with 20,000 events using 100 test particles for a physical nucleon. In the present study, nucleons are considered as being frozen out when their local densities are less than $\rho_{0} / 8$ and subsequent interactions do not cause their recapture into regions of higher density. Other emission criteria, such as taking the nucleon emission time as its last collision time in the IBUU model, do not change our conclusions. All calculated results presented in the following are in the center-of-mass system unless stated otherwise.

The EOS or the energy per particle of an asymmetric nuclear matter with density $\rho$ and isospin asymmetry $\delta=\left(\rho_{n}-\rho_{p}\right) / \rho$, where $\rho_{n}$ and $\rho_{p}$ are, respectively, its neutron and proton densities, is usually approximated by a parabolic law [12], i.e.,

$$
E(\rho, \delta)=E(\rho, 0)+E_{\text {sym }}(\rho) \delta^{2},
$$

where $E(\rho, 0)$ is the energy per particle of symmetric nuclear matter and $E_{\mathrm{sym}}(\rho)$ is the nuclear symmetry energy. For the density dependence of nuclear symmetry energy, we adopt the parameterization used in Ref. [53] for studying the properties of neutron stars, i.e.,

$$
E_{\mathrm{sym}}(\rho)=E_{\mathrm{sym}}\left(\rho_{0}\right) \cdot u^{\gamma},
$$

where $u \equiv \rho / \rho_{0}$ is the reduced density and $E_{\text {sym }}\left(\rho_{0}\right)=30$ $\mathrm{MeV}$ is the symmetry energy at normal nuclear matter density. In the present study, we consider the two cases of $\gamma=0.5$ (soft) and 2 (stiff) to explore the large range of $E_{\text {sym }}(\rho)$ predicted by many-body theories [9].

Before investigating isospin effects on light cluster production from heavy-ion collisions induced by radioactive 


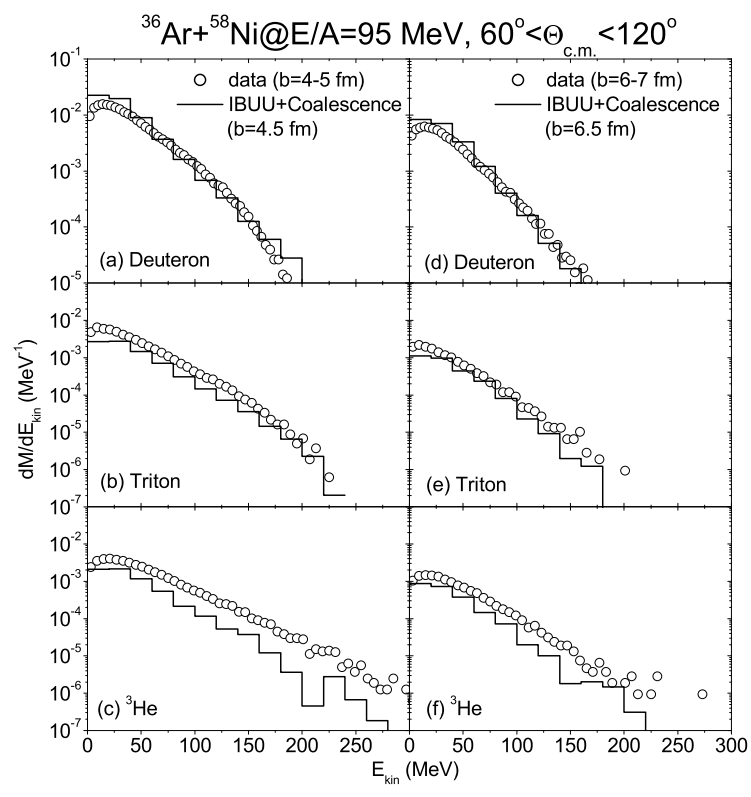

FIG. 2: Experimental (open circles) and theoretical (histogram) energy spectra of deuterons ((a) and (d)), tritons ((b) and (e)), and ${ }^{3} \mathrm{He}\left((\mathrm{c})\right.$ and (f)) from ${ }^{36} \mathrm{Ar}+{ }^{58} \mathrm{Ni}$ collisions at $E / A=95 \mathrm{MeV}$ for impact parameter $b=4.5$ (left panels) and 6.5 (right panels) fm and $\Theta_{\text {c.m. }}=60^{\circ}-120^{\circ}$.

nuclei, we first compare theoretical results obtained from the coalescence model using nucleon phases space distribution functions from the IBUU model with existing experimental data from the collisions ${ }^{36} \mathrm{Ar}+{ }^{58} \mathrm{Ni}$ at $E / A=95 \mathrm{MeV}$ at two impact parameters $b=4.5$ and 6.5 $\mathrm{fm}$. In the IBUU model simulations, we use the default parameters, i.e., the hard EOS with $K_{0}=380 \mathrm{MeV}$, the experimental free-space cross sections $\sigma_{\text {exp }}$, and the soft symmetry potential. However, the theoretical results are not corrected by experimental filters, and this may be partially responsible for their deviations from the experimental data. In Fig. 2] the energy spectra of deuteron, triton, and ${ }^{3} \mathrm{He}$ at center-of-mass angles $\Theta_{\text {c.m. }}=60^{\circ}-120^{\circ}$ are shown in Fig. 2 by solid curves for the coalescence model and by open circles for the experimental data 35. We see that the theoretical results reproduce satisfactorily the experimental deuteron energy spectra for both mid-central $(b=4.5 \mathrm{fm})$ and mid-peripheral $(b=6.5 \mathrm{fm})$ collisions except that they overestimate the yield at low kinetic energies. For the triton kinetic energy spectra, theoretical results also agree reasonably with experimental data at both impact parameters except at low kinetic energies, where they are below the measured triton yield. For ${ }^{3} \mathrm{He}$, both the yield and slope parameter of their kinetic energy spectra from theoretical calculations are somewhat smaller than experimental data. The flatter or harder energy spectra of ${ }^{3} \mathrm{He}$ than that of deuterons and tritons, known as the "3He puzzle", has been seen in other experiments as well [54, 55, [56, 57, 58, 59.

For a more quantitative comparison, we show in Table III the predicted multiplicities and average kinetic ener-
TABLE II: Calculated multiplicities and average kinetic energies per nucleon $\left(\mathrm{MeV} /\right.$ nucleon) of deuteron, triton, and ${ }^{3} \mathrm{He}$ together with the corresponding experimental data [35].

\begin{tabular}{cccccc}
\hline \hline$b(\mathrm{fm})$ & Particle & $M_{60^{\circ}-90^{\circ}}^{\text {exp }}$ & $M_{60^{\circ}-90^{\circ}}^{\text {cal }}$ & $E_{60^{\circ}-90^{\circ}}^{\text {exp }}$ & $E_{60^{\circ}-90^{\circ}}^{\text {cal }}$ \\
\hline \multirow{3}{*}{$4-5$} & $\mathrm{~d}$ & 0.814 & 1.15 & 18.2 & 15.7 \\
& $\mathrm{t}$ & 0.308 & 0.163 & 11.6 & 11.9 \\
& ${ }^{3} \mathrm{He}$ & 0.251 & 0.128 & 14.7 & 11.9 \\
\hline \multirow{3}{*}{$6-7$} & $\mathrm{~d}$ & 0.291 & 0.410 & 15.9 & 14.7 \\
& $\mathrm{t}$ & 0.090 & 0.057 & 9.8 & 10.3 \\
& ${ }^{3} \mathrm{He}$ & 0.077 & 0.044 & 12.0 & 10.4 \\
\hline \hline
\end{tabular}

gies per nucleon of deuteron, triton, and ${ }^{3} \mathrm{He}$ in center-ofmass angles $\Theta_{\text {c.m. }}=60^{\circ}-90^{\circ}$ together with corresponding experimental data. It is seen that the deuteron multiplicity from our model is about $40 \%$ above the experimental data, while those for triton and ${ }^{3} \mathrm{He}$ are about $50 \%$ below the experimental data. As to the average kinetic energy per nucleon, our model describes reasonably those of deuteron and triton but underestimates that of ${ }^{3} \mathrm{He}$. Failure of our model for ${ }^{3} \mathrm{He}$ production may be related to the neglect in the coalescence model of its binding energy, which is larger than those of deuteron and triton. Also, the harmonic oscillator wave functions used for ${ }^{3} \mathrm{He}$ may not be as good an approximation for tritons. Furthermore, in the present dynamic coalescence model, we have neglected the final-state long range Coulomb potential acting on protons after they freeze out, which would increase the kinetic energy of produced clusters, particularly ${ }^{3} \mathrm{He}$ that has a larger charge to mass ratio than deuterons and tritons. In addition, the momentumdependence of the isoscalar nuclear potential or symmetry potential, which is not considered in the present work, may change the particle energy spectra. Although these corrections need to be included for a more quantitative study, the symmetry energy effect on cluster production shown below is not expected to be appreciably affected.

\section{ISOSPIN EFFECTS ON LIGHT CLUSTER PRODUCTION}

To study isospin effects on cluster production in heavy ion collisions, we consider mainly the reaction system of ${ }^{52} \mathrm{Ca}+{ }^{48} \mathrm{Ca}$, which has an isospin asymmetry $\delta=0.2$ and can be studied at future Rare Isotope Accelerator Facility. Another heavier reaction system of ${ }^{132} \mathrm{Sn}+{ }^{124} \mathrm{Sn}$, which has a similar isospin asymmetry, will also be investigated to see how the isospin effect changes with the mass of the reaction system. In particular, we have considered the collisions ${ }^{52} \mathrm{Ca}+{ }^{48} \mathrm{Ca}$ and also ${ }^{132} \mathrm{Sn}+{ }^{124} \mathrm{Sn}$ at an incident energy of $80 \mathrm{MeV} /$ nucleon. We choose these reaction systems and incident energy as effects due to feedback from heavy fragment evaporation and feeddown from highly excited fragments to light cluster production, which cannot be described by our model, are 
expected to be less important than in collisions between heavier nuclei at lower energies.

\section{A. Yields and energy spectra of light clusters}

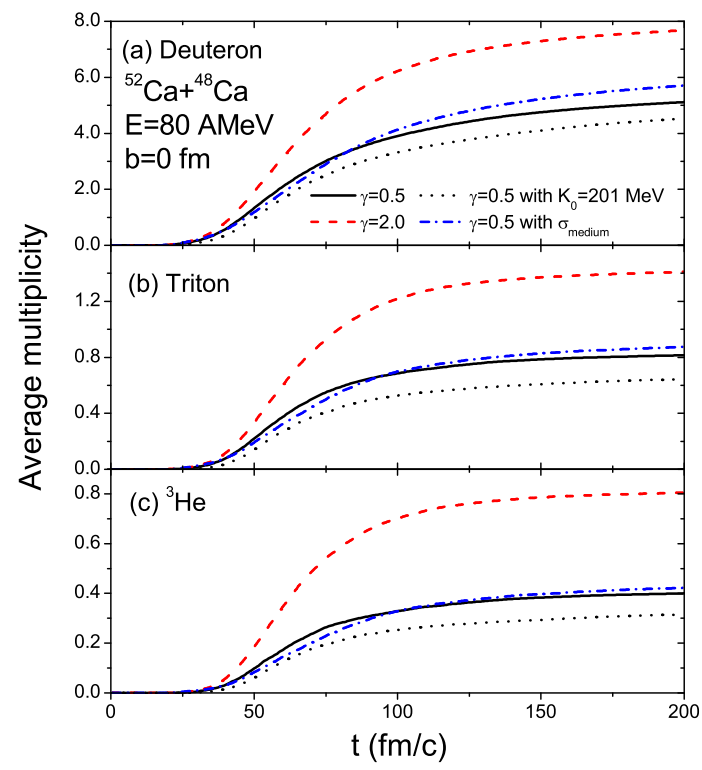

FIG. 3: Time evolution of the average multiplicities of (a) deuterons, (b) tritons, and (c) ${ }^{3} \mathrm{He}$ from central collisions of ${ }^{52} \mathrm{Ca}+{ }^{48} \mathrm{Ca}$ at $E=80 \mathrm{MeV} /$ nucleon by using the soft (solid curves) or stiff (dashed curves) symmetry energy with a stiff nuclear compressibility $K_{0}=380 \mathrm{MeV}$ and free nucleonnucleon cross sections. Results using soft symmetry energy and free nucleon-nucleon cross sections but $K_{0}=201 \mathrm{MeV}$ are shown by dotted curves, while those from soft symmetry energy and $K_{0}=380 \mathrm{MeV}$ but in-medium nucleon-nucleon cross sections are given by dash-dotted curves.

The yields and energy spectra of light clusters from heavy ion collisions are important experimental observables that can provide useful information about particle production mechanism and reaction dynamics. Shown in Figs. 3 (a), (b) and (c) is the time evolution of the average multiplicities of deuteron, triton, and ${ }^{3} \mathrm{He}$ from central collisions of ${ }^{52} \mathrm{Ca}+{ }^{48} \mathrm{Ca}$ at $E=80 \mathrm{MeV} /$ nucleon by using the soft (solid curve) or stiff (dashed curve) symmetry energy in the IBUU model. It is seen that production of these light clusters from a neutron-rich reaction system is sensitive to the density dependence of nuclear symmetry energy. The final multiplicities of deuteron, triton, and ${ }^{3} \mathrm{He}$ for the stiff symmetry energy are larger than those for the soft symmetry energy by $51 \%, 73 \%$, and $100 \%$, respectively. This is due to the fact that the stiff symmetry energy induces a stronger pressure in the reaction system and thus causes an earlier emission of neutrons and protons than in the case of the soft symmetry energy 60], leading to a stronger correlations among nucleons. Furthermore, the soft symmetry energy, which gives a more repulsive symmetry potential for neutrons and more attractive one for protons in low density region $\left(\leq \rho_{0}\right)$ than those from the stiff symmetry energy, generates a larger phase-space separation between neutrons and protons at freeze out and thus a weaker correlations among nucleons. The larger sensitivity of the multiplicity of ${ }^{3} \mathrm{He}$ to the nuclear symmetry energy than those of triton and deuteron seen in Fig. 3 reflects the fact that the effect of symmetry energy is stronger on protons than neutrons with lower momenta 60].

We have also studied the effects of using different nuclear incompressibilities and nucleon-nucleon cross sections. As shown in Fig. 3] changing the incompressibility from $K_{0}=380$ to $201 \mathrm{MeV}$ (dotted curves) or using $\sigma_{\text {medium }}$ instead of $\sigma_{\exp }$ (dash-dotted curves) only leads to a small change in the yields of light clusters. This implies that the relative space-time structure of neutrons and protons at freeze out is not sensitive to the EOS of symmetric nuclear matter and the medium dependence of nucleon-nucleon cross sections. As explained in Ref. 25], this is due to the fact that the two symmetric-matter EOS's create a similar pressure effect on particle emissions as they lead to different density evolutions with the soft one giving a larger maximum density.

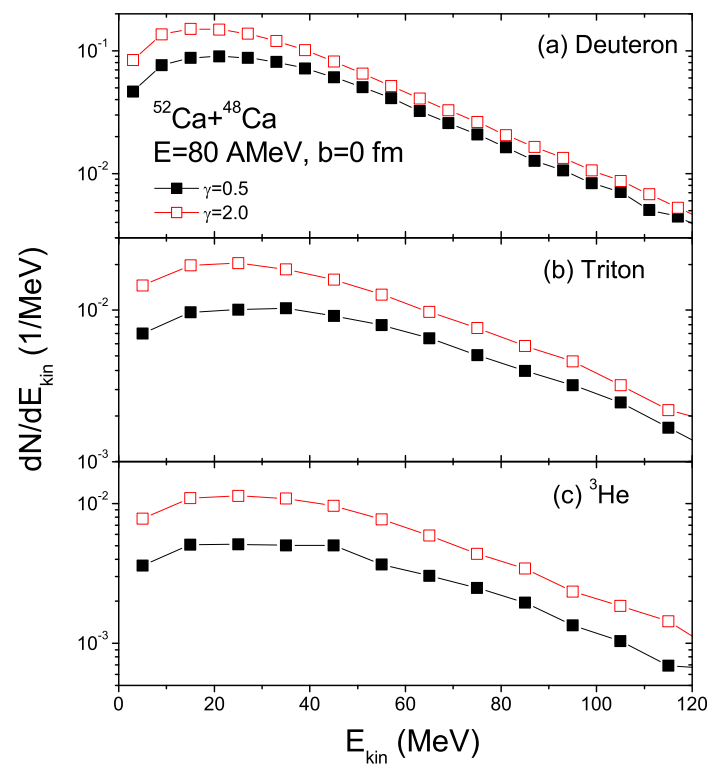

FIG. 4: Kinetic energy spectra in center-of-mass system for (a) deuterons, (b) tritons, and (c) ${ }^{3} \mathrm{He}$ from central collisions of ${ }^{52} \mathrm{Ca}+{ }^{48} \mathrm{Ca}$ at $E=80 \mathrm{MeV} /$ nucleon by using the soft (solid squares) or stiff (open squares) symmetry energy with a stiff nuclear compressibility $K_{0}=380 \mathrm{MeV}$ and free nucleon-nucleon cross sections.

The kinetic energy spectra in the center-of-mass system for deuterons, tritons, and ${ }^{3} \mathrm{He}$ 's are shown in Fig. 4 for both the soft (solid squares) and stiff (open squares) symmetry energies. It is seen that the symmetry energy has a stronger effect on the yield of low energy clusters than that of high energy ones, although the effect is still appreciable for high energy clusters. For example, the symmetry energy effect on the yield of deuteron, triton, 
and ${ }^{3} \mathrm{He}$ is about $60 \%, 100 \%$, and $120 \%$, respectively, if their kinetic energies are around $10 \mathrm{MeV}$, but is about $30 \%, 40 \%$, and $85 \%$, respectively, if their kinetic energies are around $100 \mathrm{MeV}$. This follows from the fact that lower energy clusters are emitted later in time when the size of nucleon emission source is relatively independent of nuclear symmetry energy, leading thus to similar probabilities for nucleons to form light clusters. Since there are more low energy nucleons for a stiffer symmetry energy, more light clusters are produced. On the other hand, higher energy nucleons are emitted earlier when the size of emission source is more sensitive to the symmetry energy, with a smaller size for a stiffer symmetry energy. The probability for light cluster formation is thus larger for a stiffer symmetry energy. This effect is, however, reduced by the smaller number of high energy nucleons if the symmetry energy is stiffer. As a result, production of high energy light clusters is less sensitive to the stiffness of symmetry energy. This is different from that seen in the correlation functions between two nucleons with low relative momentum, where the symmetry energy effect is larger for nucleon pairs with higher kinetic energies [25], as they are affected only by the size of emission source but not by the number of emitted nucleon pairs.

\section{B. The isobaric yield ratio $t /{ }^{3} \mathrm{He}$}

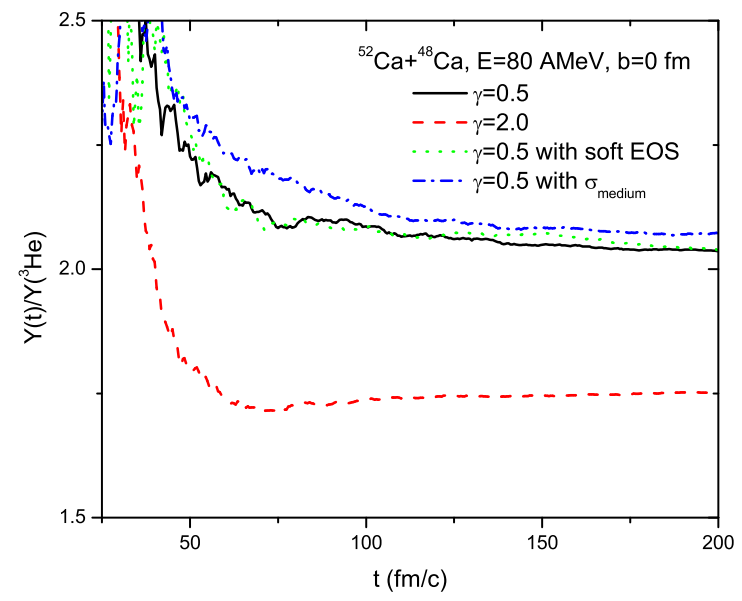

FIG. 5: Time evolution of the isobaric yield ratio $\mathrm{t} /{ }^{3} \mathrm{He}$ from central collisions of ${ }^{52} \mathrm{Ca}+{ }^{48} \mathrm{Ca}$ at $E=80 \mathrm{MeV} /$ nucleon using different symmetry energies, nuclear incompressibilities, and $N-N$ cross sections.

To minimize the model dependence and also other effects, such as the feedback from heavy fragment evaporation and the feed-down from produced excited states of triton and ${ }^{3} \mathrm{He}$, it is of interest to study the isobaric yield ratio $t /{ }^{3} \mathrm{He}$. In Fig. 5 we show the time evolution of the isobaric yield ratio $t /{ }^{3} \mathrm{He}$ from central collisions of ${ }^{52} \mathrm{Ca}$ $+{ }^{48} \mathrm{Ca}$ at $E=80 \mathrm{MeV} /$ nucleon for different symmetry energies. It is seen that the stiff symmetry energy gives a final isobaric yield ratio $\mathrm{t} /{ }^{3} \mathrm{He}$ that is about $17 \%$ less than that for the soft symmetry energy. This is due to the fact that a soft symmetry energy increases neutron emission and reduces proton emission while a stiff symmetry energy enhances the emission of both neutrons and protons. As a result, the $t /{ }^{3} \mathrm{He}$ ratio is enhanced using a soft symmetry energy but is reduced using a stiff symmetry energy. It is further seen that the effect of symmetry energy on the $t /{ }^{3} \mathrm{He}$ ratio is more pronounced at earlier stage of reactions, e.g., at $70 \mathrm{fm} / \mathrm{c}$ the $\mathrm{t} /{ }^{3} \mathrm{He}$ ratio is increased by $23 \%$ from the stiff to the soft symmetry energy.

We have also studied the effect of nuclear incompressibility and $\mathrm{N}-\mathrm{N}$ cross sections on the t/ ${ }^{3} \mathrm{He}$ ratio. As shown in Fig [ [ changing nuclear incompressibility from $K_{0}=380$ to $201 \mathrm{MeV}$ or using $\sigma_{\text {medium }}$ instead of $\sigma_{\text {exp }}$ increases the ratio $\mathrm{t} /{ }^{3} \mathrm{He}$ by less than $4 \%$. These results imply again that the relative space-time structure of neutrons and protons at freeze-out is not sensitive to the EOS of symmetric nuclear matter and the medium dependence of nucleon-nucleon cross sections.

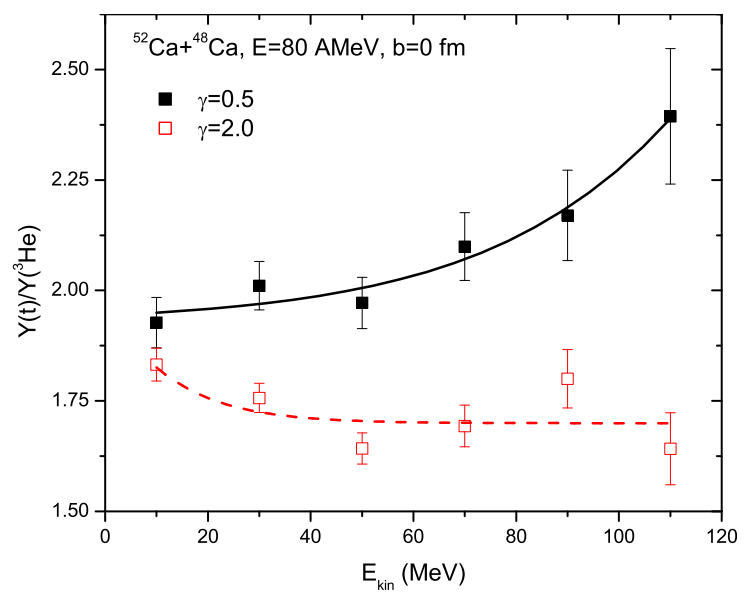

FIG. 6: The isobaric ratio $\mathrm{t} /{ }^{3} \mathrm{He}$ as a function of cluster kinetic energy in the center-of-mass system by using the soft or stiff symmetry energy. The lines are drawn to guide the eye.

To further investigate the symmetry energy effect, we show in Fig. [6 the $\mathrm{t} /{ }^{3} \mathrm{He}$ ratio with statistical errors as a function of cluster kinetic energy in the center-ofmass system for the soft (solid squares) and stiff (open squares) symmetry energies. It is seen that the ratio $\mathrm{t} /{ }^{3} \mathrm{He}$ obtained with different symmetry energies has very different energy dependence. While the $t /{ }^{3} \mathrm{He}$ ratio increases with kinetic energy for the soft symmetry energy, it decreases with kinetic energy for the stiff symmetry energy. The symmetry energy thus affects more strongly the ratio of high kinetic energy triton and ${ }^{3} \mathrm{He}$. For both soft and stiff symmetry energies, the ratio $t /{ }^{3} \mathrm{He}$ is larger than the neutron to proton ratio of the whole reaction system, i.e., $N / Z=1.5$. This is in agreement with available experimental results and also with statistical model simulations in other reaction systems and incident energies $59,61,62,63,64,65,666$. It is interesting to note that although the yield of lower energy triton and ${ }^{3} \mathrm{He}$ 
is more sensitive to symmetry energy than higher energy ones, as shown in Fig. 4 their ratio at higher energy is affected more by the symmetry energy. Moreover, the energy dependence of the ratio $\mathrm{t} /{ }^{3} \mathrm{He}$ is insensitive to the EOS of symmetric nuclear matter and the medium dependence of nucleon-nucleon cross sections. Therefore, the pre-equilibrium triton to ${ }^{3} \mathrm{He}$ ratio is a sensitive probe to the density dependence of nuclear symmetry energy.

\section{Centrality, incident energy, and reaction system dependence}

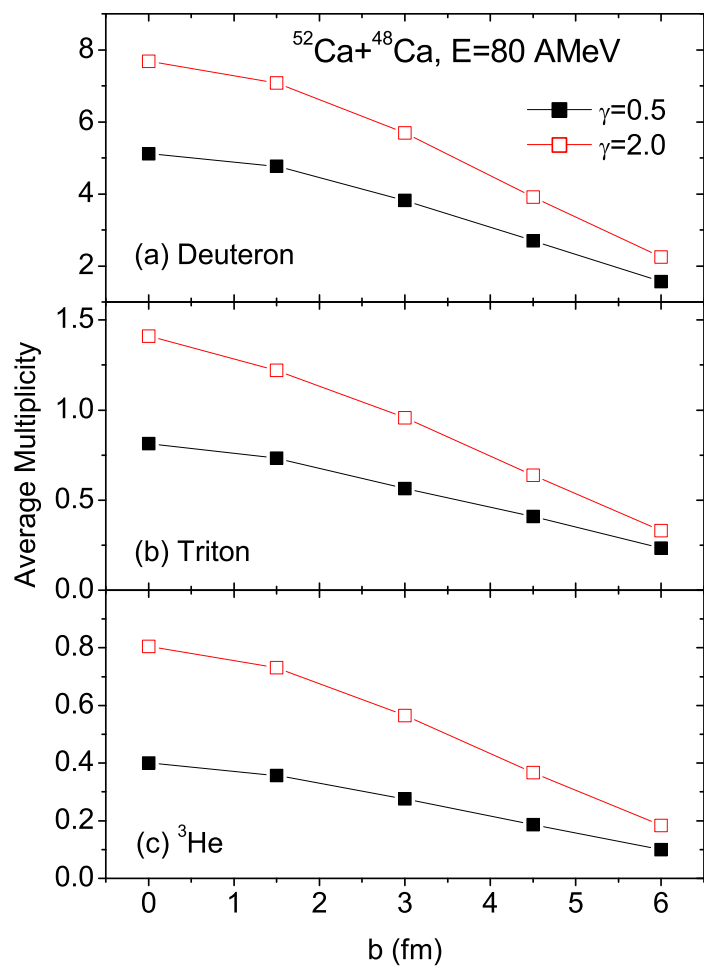

FIG. 7: Impact parameter dependence of the yield for (a) deuterons, (b) tritons, and (c) ${ }^{3} \mathrm{He}$, by using $K_{0}=380 \mathrm{MeV}$ with the soft (filled squares) or stiff (open squares) symmetry energy.

Light cluster production in heavy ion collisions also depends on the impact parameter, incident energy, and the mass of the reaction system. Fig. 7 shows the impact parameter dependence of light cluster yield by using $K_{0}=380 \mathrm{MeV}$ with the soft (filled squares) and stiff (open squares) symmetry energies. It is seen that the symmetry energy effect on the yield of light clusters decreases slightly with increasing impact parameter. This simply reflects the fact that nuclear compression becomes weaker when the impact parameter increases and the symmetry energy effect mainly comes from the difference between the soft and stiff symmetry energies at low densities. Also, we find that the light cluster yield decreases with increasing impact parameter as a result of smaller number of free nucleons in collisions at larger impact parameters.

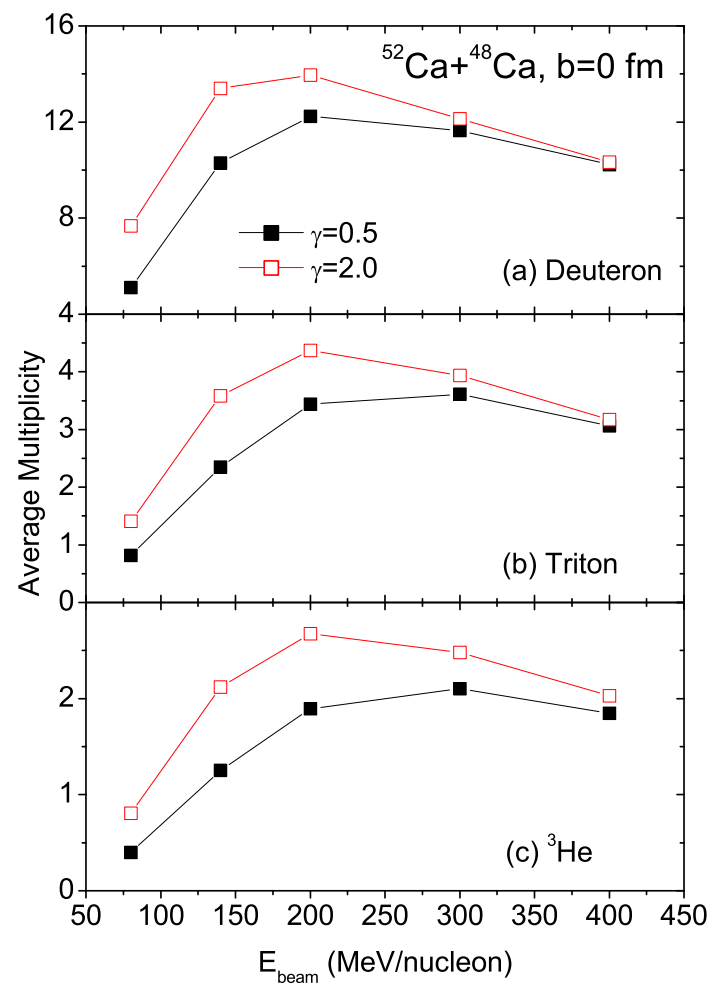

FIG. 8: Dependence of the yield for (a) deuterons, (b) tritons, and (c) ${ }^{3} \mathrm{He}$ on beam energy. The results are obtained using $K_{0}=380 \mathrm{MeV}$ with the soft (filled squares) or stiff (open squares) symmetry energy.

Dependence of the light cluster yield on the incident energy of heavy ion collisions is shown in Fig. 8. The results are obtained using $K_{0}=380 \mathrm{MeV}$ with the soft (filled squares) or stiff (open squares) symmetry energy. These results show that for the light clusters considered here their yields first increase and then decrease with increasing incident energy. The initial increase is due to increasing compression of nuclear matter with increasing incident energy. As a result, nucleons are emitted earlier and the source size for cluster production is smaller, leading thus to a larger probability for cluster production. On the other hand, with further increase in incident energy, the phase-space volume occupied by nucleons becomes larger, which reduces the formation probability of light clusters. These features have been observed in previous experiments 61]. We further see that the symmetry energy effect on light cluster production becomes weaker at higher incident energies. This is due to the fact that at higher incident energies the symmetry energy effect mainly comes from early compression stage of heavy ion collisions when the isoscalar part of nuclear equation of state dominates. Therefore, the colliding system expands rapidly at high incident energies, and the low density behavior of symmetry potential thus plays a less important role. On the other hand, the initial compression is rela- 
tively weak at lower incident energies, and the behavior of symmetry energy at low densities becomes relevant.

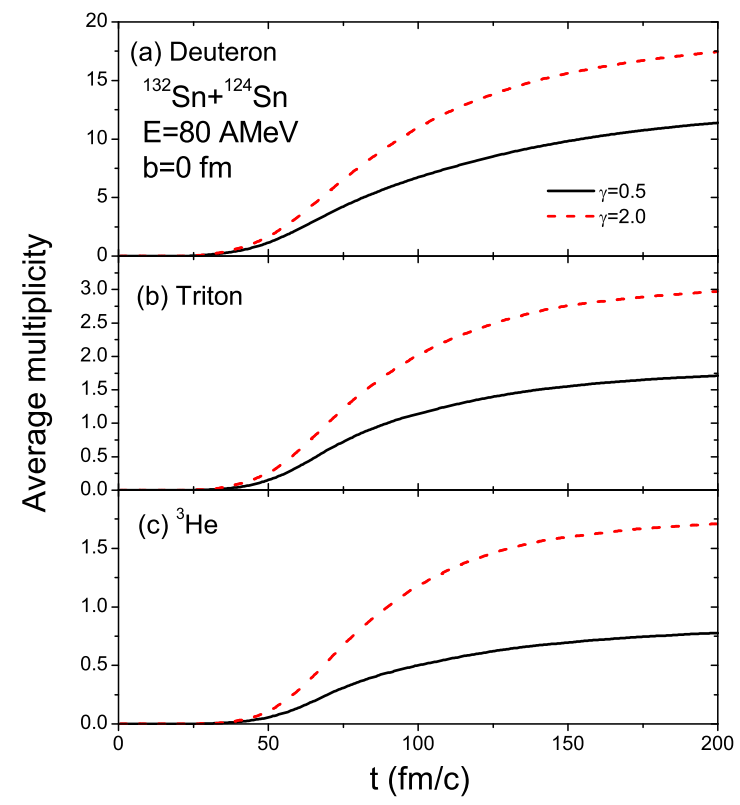

FIG. 9: Time evolution of the average multiplicity of (a) deuteron, (b) triton, and (c) ${ }^{3} \mathrm{He}$ from central collisions of ${ }^{132} \mathrm{Sn}+{ }^{124} \mathrm{Sn}$ at $80 \mathrm{MeV} /$ nucleon by using $K_{0}=380 \mathrm{MeV}$ with the soft (solid curve) or stiff (dashed curve) symmetry energy.

To see how the yield of light clusters changes in different reaction systems, we shown in Fig. 9 the time evolution of the average multiplicities of deuteron, triton, and ${ }^{3} \mathrm{He}$ from central collisions of the reaction system ${ }^{132} \mathrm{Sn}$ $+{ }^{124} \mathrm{Sn}$ at $80 \mathrm{MeV} /$ nucleon by using $K_{0}=380 \mathrm{MeV}$ with the soft (solid line) or stiff (dashed line) symmetry energy. This reaction system has a similar isospin asymmetry, i.e., $\delta=0.22$, as the reaction system ${ }^{52} \mathrm{Ca}+{ }^{48} \mathrm{Ca}$ studied in the above, and can also be studied in future RIA. It is seen that the light cluster yield in ${ }^{132} \mathrm{Sn}+$ ${ }^{124} \mathrm{Sn}$ reactions is larger than that in ${ }^{52} \mathrm{Ca}+{ }^{48} \mathrm{Ca}$ reactions shown in Fig. 3 as a result of more free neutrons in the heavier reaction system. The symmetry energy effect is slightly stronger in ${ }^{132} \mathrm{Sn}+{ }^{124} \mathrm{Sn}$ reactions than in ${ }^{52} \mathrm{Ca}+{ }^{48} \mathrm{Ca}$. Explicitly, the final multiplicities of deuteron, triton, and ${ }^{3} \mathrm{He}$ for the stiff symmetry energy are larger than those for the soft symmetry energy by $53 \%, 74 \%$, and $120 \%$, respectively.

\section{CORRELATION FUNCTIONS OF LIGHT CLUSTERS}

The particle emission function, which is important for understanding the reaction dynamics in heavy ion collisions, can be extracted from two-particle correlation functions; see, e.g., Refs. 67, 68, 69, 70] for reviews. Most extensively studied one, both experimentally and theoretically, has been the two-proton correlation function $71,72,73,74,75,76$. Recently, data on twoneutron, neutron-proton correlation functions have also become available. The neutron-proton correlation function is especially useful as it is free of correlations due to wave-function anti-symmetrization and Coulomb interactions. Indeed, Ghetti et al. have deduced from the measured neutron-proton correlation function the time sequence of neutron and proton emissions [77, 78]. The fragment-fragment correlation functions have also provided useful information about the particle production mechanism and reaction dynamics in heavy ion collisions at intermediate energies $79,80,81,82,83,84,85,86$, 87, 88].

\section{A. Average emission times of light clusters}

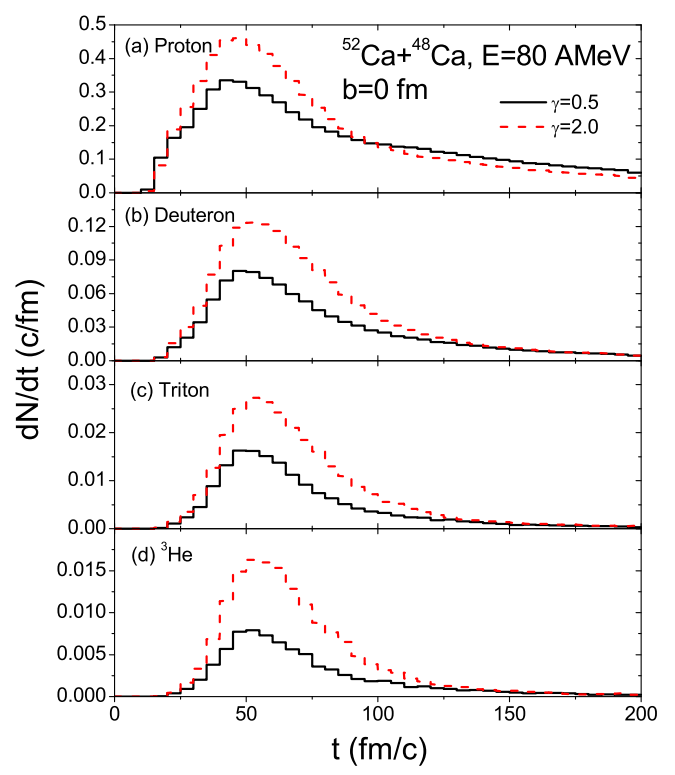

FIG. 10: Emission time distributions for (a) protons, (b) deuterons, (c) tritons, and (d) ${ }^{3} \mathrm{He}$ by using $K_{0}=380 \mathrm{MeV}$ with the soft (solid line) or stiff (dashed line) symmetry energy.

The emission times of different particles in heavy ion collisions are relevant for understanding both the collision dynamics and the mechanism of particle production. In heavy ion collisions at intermediate energies, nucleon emissions are mainly governed by the pressure of the excited nuclear matter during the initial stage of collisions [21, 89]. Since the stiff symmetry energy gives a larger pressure than that due to the soft symmetry energy [60], it leads to an earlier emission of neutrons and protons. To see the symmetry energy effect on light cluster emissions, we show in Figs. 10 (a), (b), (c) and (d) the emission time distributions for protons, deuterons, tritons, and ${ }^{3} \mathrm{He}$, respectively, by using $K_{0}=380 \mathrm{MeV}$ with the soft (solid line) or stiff (dashed line) symmetry energy. One sees 
that the emission time distributions of light clusters are different from that of protons. While the proton emission time peaks earlier at $\sim 45 \mathrm{fm} / \mathrm{c}$ and lasts longer, the emission time of light clusters peaks later at $\sim 55$ $\mathrm{fm} / \mathrm{c}$ and has a shorter duration. Light cluster production thus occurs mainly from the pre-equilibrium stage of heavy ion collisions as in previous BUU model simulations 83]. As to the symmetry energy effect on particle emissions, it is seen from Fig. 10 that the particle emission times are earlier for the stiff symmetry energy than for the soft symmetry energy as the former gives a larger initial pressure.

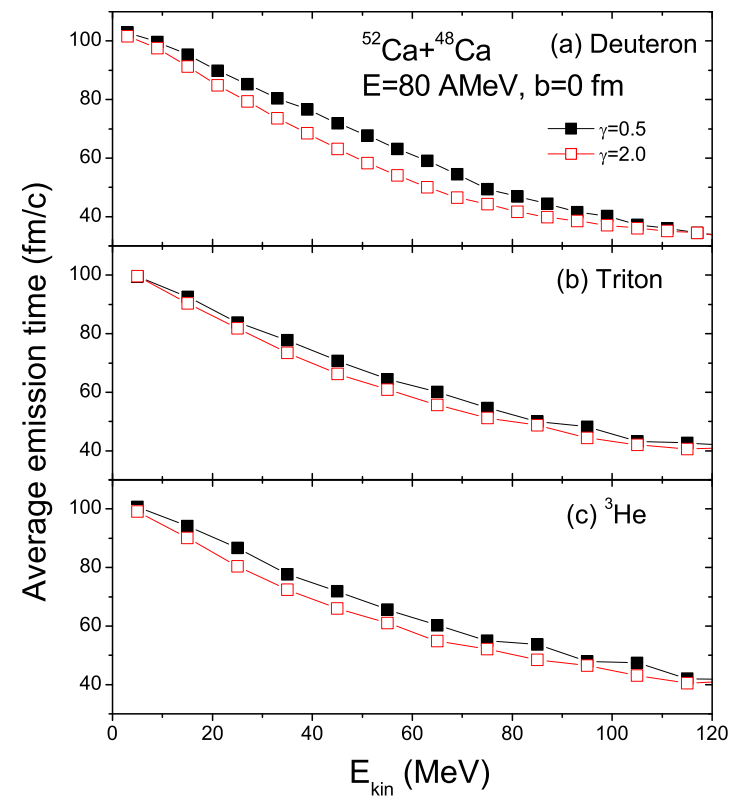

FIG. 11: Average emission times of (a) deuterons, (b) tritons, and (c) ${ }^{3} \mathrm{He}$ as functions of their c.m. kinetic energies by using $K_{0}=380 \mathrm{MeV}$ with the soft (filled squares) or stiff symmetry energy (open squares).

Particles emitted in earlier stage of heavy ion collisions usually have higher energy than those emitted during later stage of the reaction. It is thus of interest to study the correlation between the emission time of a particle and its energy. Shown in Fig. 11] are the average emission times of deuterons, tritons, and ${ }^{3} \mathrm{He}$ as functions of their c.m. kinetic energies by using $K_{0}=380$ $\mathrm{MeV}$ with the soft or stiff symmetry energy. It is seen that the average emission time of a particle with a given c.m. kinetic energy is earlier for the stiff symmetry energy (open squares) than for the soft one (filled squares). These features are consistent with results shown in Fig. 10] on the particle emission time distributions. It is also worth noting that the emission time of a particle also depends on its energy, i.e., particles with higher kinetic energies are emitted earlier than those with lower kinetic energies.

To see the effect of symmetry energy on the emission sequence of different particles, we show in Fig. 12 the average emission times of neutrons, protons, deuterons,

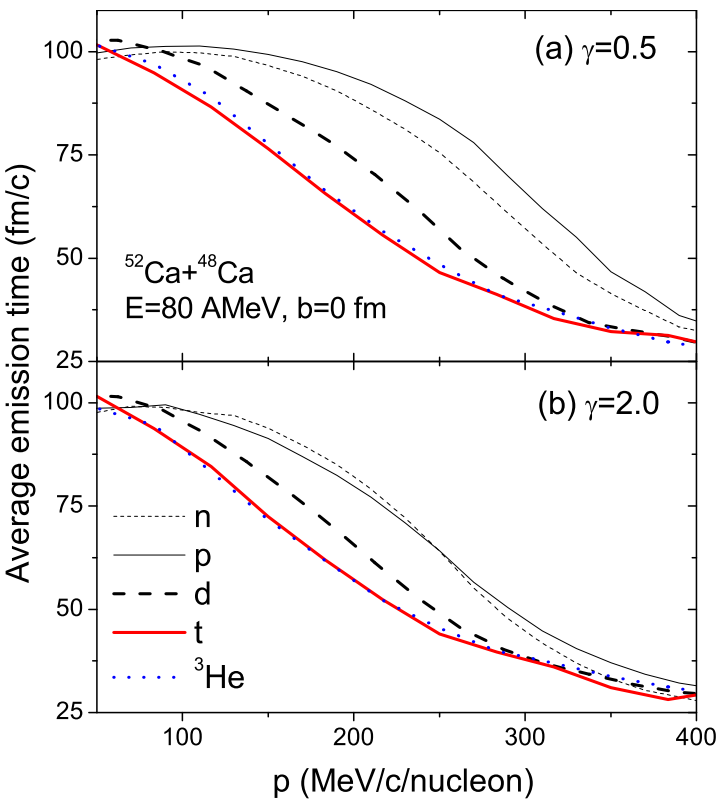

FIG. 12: Average emission times of neutrons, protons, deuterons, tritons, and ${ }^{3} \mathrm{He}$ as functions of their momenta per nucleon by using $K_{0}=380 \mathrm{MeV}$ with the soft (upper panel) or stiff (lower panel) symmetry energy.

tritons, and ${ }^{3} \mathrm{He}$ as functions of their momenta per nucleon by using $K_{0}=380 \mathrm{MeV}$ with the soft (upper panel) or stiff (lower panel) symmetry energy. It is seen that ordering of the average emission times for neutrons and protons depends on the stiffness of symmetry energy, with a significant delay of proton emission in the case of soft symmetry energy. The ordering of average emission times for light clusters depends further on their masses, with heavier clusters emitted earlier than lighter ones at the same momentum per nucleon, which is expected from the coalescence model. We note that although the density dependence of nuclear symmetry energy influences significantly the ordering of neutron and proton average emission times, it has only a weak effect on that for tritons and ${ }^{3} \mathrm{He}$. Experimentally, the particle emission sequence can be studied from the correlation functions of non-identical particles using various energy cuts [77, 78, 88, 90].

\section{B. Correlation functions of deuteron-deuteron, triton-triton, and ${ }^{3} \mathrm{He}-{ }^{3} \mathrm{He}$}

In standard Koonin-Pratt formalism [91, 92, 93], the two-particle correlation function is obtained by convoluting the emission function $g(\mathbf{p}, x)$, i.e., the probability for emitting a particle with momentum $\mathbf{p}$ from the spacetime point $x=(\mathbf{r}, t)$, with the relative wave function of the two interacting particles, i.e.,

$$
C(\mathbf{P}, \mathbf{q})=\frac{\int d^{4} x_{1} d^{4} x_{2} g\left(\mathbf{P} / 2, x_{1}\right) g\left(\mathbf{P} / 2, x_{2}\right)|\phi(\mathbf{q}, \mathbf{r})|^{2}}{\int d^{4} x_{1} g\left(\mathbf{P} / 2, x_{1}\right) \int d^{4} x_{2} g\left(\mathbf{P} / 2, x_{2}\right)} .
$$


In the above, $\mathbf{P}\left(=\mathbf{p}_{\mathbf{1}}+\mathbf{p}_{\mathbf{2}}\right)$ and $\mathbf{q}\left(=\frac{1}{2}\left(\mathbf{p}_{\mathbf{1}}-\mathbf{p}_{\mathbf{2}}\right)\right)$ are, respectively, the total and relative momenta of the particle pair; and $\phi(\mathbf{q}, \mathbf{r})$ is the relative two-particle wave function with $\mathbf{r}$ being their relative position, i.e., $\mathbf{r}=\left(\mathbf{r}_{2}-\mathbf{r}_{1}\right)-$ $\frac{1}{2}\left(\mathbf{v}_{\mathbf{1}}+\mathbf{v}_{\mathbf{2}}\right)\left(t_{2}-t_{1}\right)$. This approach has been shown to be very useful in studying the reaction dynamics of intermediate energy heavy-ion collisions 68]. In the present paper, we use the Koonin-Pratt method to determine the particle-particle correlation functions in order to study the effect due to the density dependence of nuclear symmetry energy on the spatial and temporal structure of the emission source for light clusters in intermediate energy heavy ion collisions.

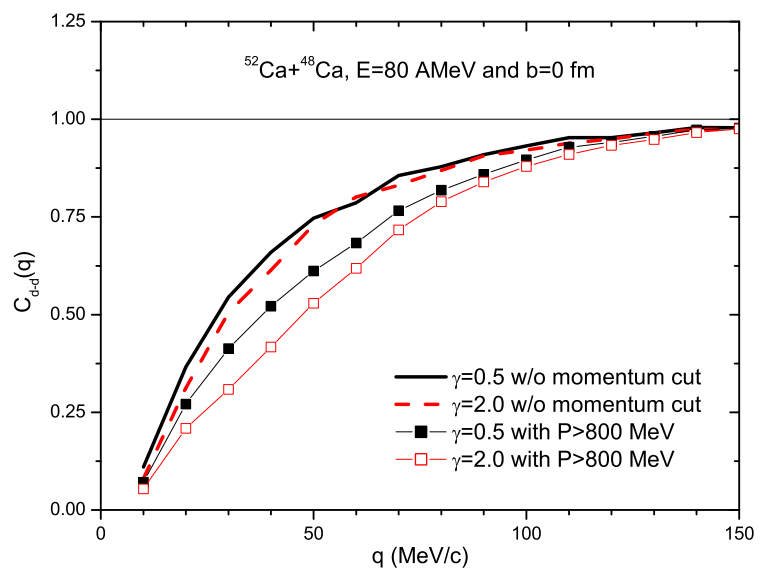

FIG. 13: Two-deuteron correlation functions from central collisions of ${ }^{52} \mathrm{Ca}+{ }^{48} \mathrm{Ca}$ at $E=80 \mathrm{MeV} /$ nucleon. Results without momentum cut are shown by solid and dashed curves for the soft and stiff symmetry energies, respectively. Curves with filled and open squares are corresponding ones for deuterons with total momentum $P>800 \mathrm{MeV} / \mathrm{c}$.

Using the program Correlation After Burner [94], which takes into account final-state particle-particle interactions, we have evaluated two-cluster correlation functions from the emission function given by the IBUU model. We first show in Fig. 13 the two-deuteron correlation functions from central collisions of ${ }^{52} \mathrm{Ca}+{ }^{48} \mathrm{Ca}$ at $E=80 \mathrm{MeV} /$ nucleon. Solid and dashed curves are results without momentum cut using the soft and stiff symmetry energies, respectively. The results for deuteron pairs with total momenta $P>800 \mathrm{MeV} / \mathrm{c}$ are shown, respectively, by curves with filled and open squares for the soft and stiff symmetry energies. In obtaining these results, the final-state strong interaction between two deuterons includes only the relative $s$-wave interaction, which we use the one in Ref. 67 that fits the deuterondeuteron elastic scattering phase-shift by Woods-Saxon potential. Because of the repulsive $s$-wave nuclear potential and Coulomb potential, two deuterons are anticorrelated as shown in Fig. 13] and the anti-correlation is stronger for deuteron pairs with total momenta $P>800$ $\mathrm{MeV} / \mathrm{c}$. The latter implies that deuterons with higher momenta are emitted earlier and thus have a smaller emission source size. Although the correlation function for all deuterons depends weakly on the stiffness of nuclear symmetry energy, that for deuterons with total momenta $P>800 \mathrm{MeV} / \mathrm{c}$ clearly shows a stronger anticorrelation for the stiff than for the soft symmetry energy. For example, the soft symmetry energy gives a correlation function at $q=40 \mathrm{MeV} / \mathrm{c}$ that is $20 \%$ larger than that due to the stiff symmetry energy. Therefore, preequilibrium deuterons with higher momenta are emitted earlier or have a smaller source size for the stiff symmetry energy, consistent with the results shown in Fig. [11 on their average emission times. The correlation function of deuteron pairs with high total momenta is thus sensitive to the density dependence of nuclear symmetry energy.

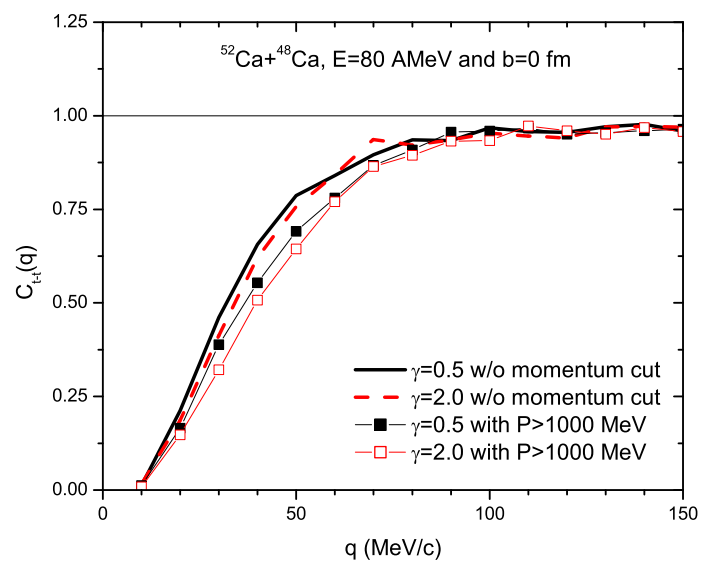

FIG. 14: Same as Fig. 13 for two-triton correlation function with and without momentum cut.

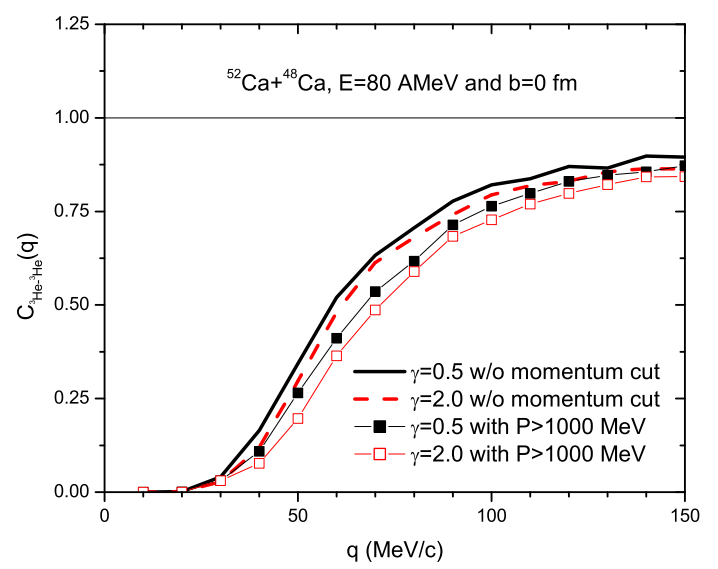

FIG. 15: Same as Fig. 13 for two- ${ }^{3} \mathrm{He}$ correlation function with and without momentum cut.

In Figs. 14 and 15 we show, respectively, the twotriton and two- ${ }^{3} \mathrm{He}$ correlation functions with and without momentum cut. Since the strong interaction potential between two tritons or ${ }^{3} \mathrm{He}$ is poorly known, the results obtained here include only the Coulomb potential in the final-state interaction as in Ref. 67]. It is seen that the stiff symmetry energy leads to a slightly stronger 
anti-correlation of triton or ${ }^{3} \mathrm{He}$ pairs than that obtained with the soft symmetry energy, particularly for triton or ${ }^{3} \mathrm{He}$ pairs with total momenta $P>1000 \mathrm{MeV} / \mathrm{c}$. The symmetry energy effect on heavier clusters is thus weaker than on lighter particles such as nucleons and deuterons, and this is consistent with the fact that the symmetry energy effect on cluster emission times becomes weaker with increasing particle mass as shown in Fig. [1]

\section{SUMMARY AND OUTLOOK}

Using an isospin-dependent transport model, we have studied light cluster production via the coalescence model in heavy-ion collisions induced by neutron-rich nuclei. We find that the density dependence of nuclear symmetry energy affects significantly the yield of deuterons, tritons, and ${ }^{3} \mathrm{He}$ in these collisions. More light clusters are produced for the stiff symmetry energy than the soft symmetry energy. This effect is particularly large when these clusters have lower kinetic energies. Also, the isobaric ratio $t /{ }^{3} \mathrm{He}$, especially for higher energy tritons and ${ }^{3} \mathrm{He}$, shows a strong sensitivity to the density dependence of nuclear symmetry energy. It is further found that light cluster production is insensitive to the EOS of the isospin-independent part of nuclear equation of state and the medium dependence of nucleon-nucleon cross sections.

We have also studied the dependence of light cluster production on the impact parameter and incident energy of heavy ion collisions as well as the mass of the reaction system. We find that the symmetry energy effect on light cluster production depends weakly on impact parameter and the mass of the reaction system. However, with increasing incident energy the symmetry energy effect on light cluster production becomes weaker.

We have further studied the emission time and sequence as well as the correlation functions of light clusters. It is found that at same momentum per nucleon heavier clusters are emitted earlier than lighter ones as expected from the coalescence model. Since the emission time of light clusters is earlier for the stiff symmetry energy than for the soft one, a stronger anti-correlation of light clusters is obtained with the stiff symmetry energy. However, the symmetry energy effect on the correlation functions of light clusters becomes weaker with increasing cluster mass.

Isospin effects on cluster production and isotopic ratios in heavy ion collisions have been previously studied using either the lattice gas model [65] or a hybrid of IBUU and statistical fragmentation model [18. These studies are, however, at lower energies than considered here, where effects due to multifragmentation as a result of possible gas-liquid phase transition may play an important role.
Except deuterons, both tritons and ${ }^{3} \mathrm{He}$ are only about one per event in heavy ion collisions at $80 \mathrm{AMeV}$. The number of other clusters such as the alpha particle is not large either [95, 96]. In this case, the coalescence model is expected to be a reasonable model for determining the production of light clusters from heavy ion collisions as demonstrated in Section III This is particularly so for light clusters with large kinetic energies as they are not contaminated by contributions from decays of heavy fragments, which are mainly of low kinetic energies and may not be negligible in intermediate energy heavy ion collisions. Furthermore, the effect obtained in present study will be enhanced if other clusters are emitted earlier during the collisions, leading to a larger isospin asymmetry in the remaining emission source for light clusters. Studies of light clusters production and correlations in heavy ion collisions induced by neutron-rich nuclei thus provide another possible tool for extracting useful information about the density-dependence of nuclear symmetry energy.

In the present work, we have not included momentum dependence in either the isoscalar mean-field potential or the symmetry potential. The former may affect the properties of nuclear emission source as it has been shown that a momentum-dependent mean-field potential reduces nuclear stopping or increases nuclear transparency [97]. With momentum dependence included in the nuclear symmetry potential, differences between the neutron and proton potentials are affected not only by the density dependence of nuclear symmetry energy but also by the magnitude of proton and neutron momenta [98, 99]. Since the momentum-dependent symmetry potential has been shown to reduce significantly the $n / p$ ratio at higher kinetic energies [100], it is expected to also affect our results on the kinetic energy dependence of $t /{ }^{3} \mathrm{He}$ ratio. It is thus of interest to study quantitatively how the results obtained in the present study are modified by the momentum dependence of nuclear meanfield potentials.

\section{Acknowledgments}

We thank Yu-Gang Ma and Joe Natowitz for discussions on light cluster production in heavy ion collisions, P. Pawlowski for providing us the experimental data, and G. Verde for discussions on light cluster correlation functions. This paper was based on work supported by the U.S. National Science Foundation under Grant Nos. PHY-0098805 and PHY-0088934 as well as the Welch Foundation under Grant No. A-1358. LWC is also supported by the National Natural Science Foundation of China under Grant No. 10105008. 
[2] K. Pomorski and J. Dudek, Phys. Rev. C 67, 044316 (2003); nucl-th/0205011

[3] Isospin Physics in Heavy-Ion Collisions at Intermediate Energies, Eds. Bao-An Li and W. Udo Schröder (Nova Science Publishers, Inc, New York, 2001).

[4] K. Oyamatsu, I. Tanihata, Y. Sugahara, K. Sumiyoshi, and H. Toki, Nucl. Phys. A634, 3 (1998).

[5] B.A. Brown, Phys. Rev. Lett. 85, 5296 (2000).

[6] C.J. Horowitz, and J. Piekarewicz, Phys. Rev. Lett 86, 5647 (2001); Phys Rev. C 63, 025501 (2001).

[7] R.J. Furnstahl, Nucl. Phys. A706, 85 (2002).

[8] H.A. Bethe, Rev. Mod. Phys. 62, 801 (1990).

[9] I. Bombaci, in [3] , p.35.

[10] J.M. Lattimer and M. Prakash, Astr. Phys. Jour. 550, 426 (2001).

[11] Radioactive Nuclear Beams, a special volume of Nucl. Phys. A693, (2001), Ed. I. Tanihata.

[12] B.A. Li, C.M. Ko, and W. Bauer, Int. Jour. Phys. E 7, 147 (1998).

[13] M. Di Toro, V. Baran, M. Colonna, G. Fabbri, A. B. Larionov, S. Maccarone, and S. Scalone, Prog. Part. Nucl. Phys. 42, 125 (1999).

[14] B.A. Li, C.M. Ko, and Z.Z. Ren, Phys. Rev. Lett. 78, 1644 (1997).

[15] B.A. Li and C.M. Ko, Nucl. Phys. A618, 498 (1997).

[16] V. Baran, M. Colonna, M. Di Toro, and A.B. Larionov, Nucl. Phys. A632, 287 (1998).

[17] H.S. Xu et al., Phys. Rev. Lett. 85, 716 (2000).

[18] W.P. Tan et al., Phys. Rev. C 64, 051901(R) (2001).

[19] V. Baran, M. Colonna, M. Di Toro, V. Greco, and M. Zielinska-Pfabé, and H.H. Wolter, Nucl. Phys. A703, 603 (2002).

[20] M.B. Tsang et al., Phys. Rev. Lett. 86, 5023 (2001).

[21] B.A. Li, A.T. Sustich, and B. Zhang, Phys. Rev. C 64, 054604 (2001).

[22] B.A. Li, Phys. Rev. Lett. 85, 4221 (2000).

[23] V. Greco, V. Baran, M. Colonna, M. Di Toro, T. Gaitanos, H.H. Wolter, Phys. Lett. B562, 215 (2003); nucl-th/0212102

[24] B.A. Li, Phys. Rev. Lett. 88, 192701 (2002); Nucl. Phys. A708, 365 (2002).

[25] L.W. Chen, V. Greco, C.M. Ko, and B.A. Li, Phys. Rev. Lett. 90, 162701 (2003); nucl-th/0211002

[26] S. Mrowczynski, Phys. Lett. B277, 43 (1992).

[27] L.W. Chen, C.M. Ko, and B.A. Li, Phys. Rev. C 68, 017601 (2003); nucl-th/0302068

[28] P.E. Hodgson and E. Bĕták, Phys. Rep. 374, 1 (2003); and the references therein.

[29] L.P. Csernai and J.I. Kapusta, Phys. Rep. 131, 223 (1986); and the references therein.

[30] M. Gyulassy, K. Frankel, and E.A. Relmer, Nucl. Phys. A402, 596 (1983).

[31] J. Aichelin, A. Rosenhauer, G. Peilert, H. Stöcker, and W. Greiner, Phys. Rev. Lett. 58, 1926 (1987).

[32] V. Koch et al., Phys. Lett. B 241, 174 (1990).

[33] R. Mattiello et al., Phys. Rev. Lett. 742180 (1995); R. Mattiello et al., Phys. Rev. C 55, 1443 (1997).

[34] J. L. Nagle et al., Phys. Rev. C 53, 367 (1996).

[35] P. Pawlowski et al., Eur. Phys. Jour. A 9, 371 (2000).

[36] S.T. Butler and C.A. Pearson, Phys. Rev. 129, 836 (1963).

[37] A. Schwarzschild and C. Zupancic, Phys. Rev. 129, 854 (1963).

[38] H.H. Gutbrod et al., Phys. Rev. Lett. 37, 667 (1976).
[39] H. Sato and K. Yazak, Phys. Lett. B 98, 153 (1981).

[40] A.Z. Mekjian, Phys. Rev. C 17, 1051 (1978); S. Das Gupta and A.Z. Mekjian, Phys. Rep. 72, 131 (1981)

[41] R. Scheibl and U. Heinz, Phys. Rev. C 59, 1585 (1999).

[42] A. Polleri et al., Nucl. Phys. A661, 452c (1999).

[43] P. E. Hodgson, Nuclear Reaction and Nuclear Structure (Clarendon, Oxford, 1971), p. 453.

[44] T. Ericsson and W. Weise, Pions and Nuclei (Clarendon, Oxford, 1988).

[45] G.R. Shin and J. Rafelski, J. Phys. G 16, L187 (1990).

[46] A.T. M. Aerts and C.B. Dover, Phys. Rev. D 28, 450 (1983).

[47] C.R. Chen, G.L. Payne, J.L. Friar, and B.F. Gibson, Phys. Rev. C 33, 1740 (1986).

[48] B.A. Li, Z.Z. Ren, C.M. Ko, and S.J. Yennello, Phys. Rev. Lett. 76, 4492 (1996); B.A. Li and A.T. Sustich, ibid. 82, 5004 (1999).

[49] G.Q. Li and R. Machleidt, Phys. Rev. C 48, 1702 (1993); ibid. 49 (1994) 566.

[50] Q. Pan and P. Danielewicz, Phys. Rev. Lett. 70, 2062 (1993).

[51] J. Zhang, S. Das Gupta, and C. Gale, Phys. Rev. C 50, 1617 (1994)

[52] G.F. Bertsch and S. Das Gupta, Phys. Rep. 160, 189 (1988).

[53] H. Heiselberg and M. Hjorth-Jensen, Phys. Rep. 328, 237 (2000).

[54] A.M. Poskanzer, G.W. Butler, and E.K. Hyde, Phys. Rev. C 3, 882 (1971).

[55] M.A. Lisa et al., Phys. Rev. Lett. 75, 2662 (1995).

[56] G. Poggi et al., Nucl. Phys. A586, 755 (1995).

[57] N. Marie et al., Phys. Lett. B391, 15 (1997).

[58] V.E. Viola, K. Kwiatkowsky, and W.A. Friedman, Phys. Rev. C 59, 2660 (1999).

[59] K. Hagel et al., Phys. Rev. C 62, 034607 (2000).

[60] L.W. Chen, V. Greco, C.M. Ko, and B.A. Li, Phys. Rev. C 68, 014605 (2003); nucl-th/0305036

[61] S. Nagamiya et al., Phys. Rev. C 24, 971 (1981).

[62] J. Cibor et al., Phys. Lett. B 473, 29 (2000).

[63] L.G. Sobotka, R.J. Charity, and J.F. Dempsey, in [3], p.331.

[64] M. Veselsky et al., Phys. Lett. B 497, 1 (2001).

[65] Ph. Chomaz and F. Gulminelli, Phys. Lett. B 447, 221 (1999).

[66] S. Das Gupta and S.K. Samaddar, in 3], p.109.

[67] D.H. Boal, C.K. Gelbke and B.K. Jennings, Rev. Mod. Phys. 62, 553 (1990).

[68] W. Bauer, C.K. Gelbke, and S. Pratt, Ann. Rev. Nucl. Part. Sci. 42, 77 (1992).

[69] D. Ardouin, Int. Jour. Phys. E 6, 391 (1997).

[70] U.A. Wiedemann and U. Heinz, Phys. Rep. 319, 145 (1999).

[71] W. G. Gong et al., Phys. Rev. Lett. 65, 2114 (1990).

[72] W.G. Gong, W. Bauer, C.K. Gelbke, and S. Pratt, Phys. Rev. C 43, 781 (1991).

[73] G. J. Kunde et al., Phys. Rev. Lett. 70, 2545 (1993).

[74] D.O. Handzy et al., Phys. Rev. Lett. 75, 2916 (1995).

[75] G. Verde, D.A. Brown, P. Danielewicz, C.K. Gelbke, W.G. Lynch, and M.B. Tsang, Phys. Rev. C 65, 054609 (2002).

[76] G. Verde, P. Danielewicz, D.A. Brown, W.G. Lynch, C.K. Gelbke, and M.B. Tsang, Phys. Rev. C 67, 034606 (2003)

[77] R. Ghetti et al., Nucl. Phys. A674, 277 (2000). 
[78] R. Ghetti et al., Phys. Rev. Lett. 87, 102701 (2001).

[79] C.B. Chitwood et al., Phys. Rev. Lett. 54, 302 (1985).

[80] J. Pochodzalla et al., Phys. Rev. Lett. 55, 177 (1985); J. Pochodzalla et al., Phys. Lett. B174, 36 (1986); J. Pochodzalla et al., Phys. Rev. C35, 1695 (1987).

[81] R.A. Kryger et al., Phys. Rev. Lett. 65, 2118 (1990); R.A. Kryger et al., Phys. Rev. C 46, 1887 (1992).

[82] F. Zhu et al., Phys. Rev. C 44, R582 (1991).

[83] W.G. Gong et al., Phys. Rev. C 47, R429 (1993).

[84] B. Erazmus, L. Martin, R. Lednicky, and N. Carjan, Phys. Rev. C 49, 349 (1994).

[85] T.M. Hamilton et al., Phys. Rev. C 53, 2273 (1996).

[86] R. Kotte et al. (The FOPI Collaboration), Eur. Phys. J. A 6, 185 (1999).

[87] D. Gourio et al., Eur. Phys. J. A 7, 245 (2000).

[88] R. Ghetti et al., in XL Int. Winter Meeting on Nucl. Phys., Bormio (Italy), 27 Jan-2 Feb, 2003

[89] P. Danielewicz, Nucl. Phys. A685, 368c (2001).
[90] C.J. Gelderloos et al., Phys. Rev. Lett. 75, 3082 (1995); C.J. Gelderloos et al., Phys. Rev. C 52, R2834 (1995)

[91] S.E. Koonin, Phys. Lett. B 70, 43 (1977).

[92] S. Pratt, Phys. Rev. Lett. 53, 1219 (1984); Phys. Rev. D33, 72 (1986).

[93] S. Pratt and M.B. Tsang, Phys. Rev. C 36, 2390 (1987).

[94] S. Pratt, Nucl. Phys. A566, 103c (1994).

[95] B.V. Jacak et al., Phys. Rev. C 35, 1751 (1987).

[96] B. Borderie et al., Phys. Lett. B388, 224 (1996).

[97] V. Greco, A. Guarnera, M. Colonna, and M. Di Toro, Phys. Rev. C 59, 810 (1999).

[98] C. B. Das, S. Das Gupta, C. Gale, and B.A. Li, Phys. Rev. C 67, 034611 (2003).

[99] A. Ono, Prog. Theor. Phys. Suppl. 146, 378 (2002).

[100] B. A. Li, C. B. Das, S. Das Gupta, and C. Gale, submitted for publication. 